\title{
Diacronie
}

Studi di Storia Contemporanea

$N^{\circ} 31,3 \mid 2017$

"Le armi della politica, la politica delle armi"

\section{Marco Di Maggio (a cura di), Sfumature di rosso. La Rivoluzione russa nella politica italiana del Novecento}

\section{Andrea Ricciardi}

\section{Q OpenEdition}

\section{Edizione digitale}

URL: http://journals.openedition.org/diacronie/6293

DOI: $10.4000 /$ diacronie.6293

ISSN: 2038-0925

\section{Editore}

Association culturelle Diacronie

Notizia bibliografica digitale

Andrea Ricciardi, « Marco Di Maggio (a cura di), Sfumature di rosso. La Rivoluzione russa nella politica italiana del Novecento », Diacronie [Online], № 31, 3 | 2017, documento 6, Messo online il 29 octobre 2017, consultato il 24 septembre 2020. URL : http://journals.openedition.org/diacronie/6293; DOI : https://doi.org/10.4000/diacronie.6293 


\title{
Diacronie
}

Studi di Storia Contemporanea

$31,3 / 2017$

"Le armi della politica, la politica delle armi". Ideologie di lotta ed esperienze di guerra

\section{RECENSIONE: Marco DI MAGGIO (a cura di), Sfumature di rosso. La Rivoluzione russa nella politica italiana del Novecento, Torino, Accademia University Press, 2017, 328 pp.}

\author{
A cura di Andrea RICCIARDI
}

Per citare questo articolo:

RICCIARDI, Andrea, «Marco DI MAGGIO (a cura di), Sfumature di rosso. La Rivoluzione russa nella politica italiana del Novecento, Torino, Accademia University Press, 2017, 328 pp.», Diacronie. Studi di Storia Contemporanea : "Le armi della politica, la politica delle armi". Ideologie di lotta ed esperienze di guerra, 31, 3/2017, 29/10/2017,

URL: < http://www.studistorici.com/2017/10/29/ricciardi_numero_31/ >

Diacronie Studi di Storia Contemporanea $\rightarrow$ http://www.diacronie.it Rivista storica online. Uscita trimestrale.

redazione.diacronie@hotmail.it

Comitato di direzione: Naor Ben-Yehoyada - João Fábio Bertonha - Christopher Denis-Delacour - Maximiliano Fuentes Codera Anders Granås Kjøstvedt - John Paul Newman - Deborah Paci - Niccolò Pianciola - Spyridon Ploumidis - Wilko Graf Von Hardenberg

Comitato di redazione: Jacopo Bassi - Luca Bufarale - Gianluca Canè - Fausto Pietrancosta - Alessandro Salvador - Matteo Tomasoni Diritti: gli articoli di Diacronie. Studi di Storia Contemporanea sono pubblicati sotto licenza Creative Commons 3.0. Possono essere riprodotti e modificati a patto di indicare eventuali modifiche dei contenuti, di riconoscere la paternità dell'opera e di condividerla allo stesso modo. La citazione di estratti è comunque sempre autorizzata, nei limiti previsti dalla legge. 


\title{
6/ Marco DI MAGGIO (a cura di), Sfumature di rosso. La Rivoluzione russa nella politica italiana del Novecento, Torino, Accademia University Press, 2017, 328 pp.
}

\author{
A cura di Andrea RICCIARDI
}

Sosteneva Vittorio Foa, nel 2006, a proposito della rivoluzione:

È da un po' di tempo che rifletto sulla rivoluzione e, più precisamente, sulla nostalgia della rivoluzione. Il Novecento è stato un secolo caratterizzato da conflitti devastanti ma anche da processi rivoluzionari, il più importante e condizionante dei quali è stato senza dubbio la Rivoluzione d'Ottobre. Oggi possiamo ragionare a freddo sul 1917, comprendiamo quanto tragica sia stata l'esperienza del comunismo non solo in URSS. Eppure, in fondo, mi sembra che sia ancora diffusa una certa nostalgia del sogno rivoluzionario. Ancora più precisamente potrei dire che si tratta della nostalgia della fattibilità della rivoluzione, cioè per l'idea che una rivoluzione sia possibile, non per la rivoluzione in sé e per i suoi effetti concreti. Mi sono detto che forse questa è semplicemente una riflessione da vecchio, propria di chi ha attraversato tutto il Novecento, con qualche appendice nel nuovo millennio, e proietta sugli altri un pensiero legato alla sua personale esperienza. Ma forse non è proprio così ${ }^{1}$.

Foa aveva probabilmente ragione e, forse, la sua riflessione vale anche per il presente, almeno se si considerano alcuni scritti elaborati sul 1917 a cento anni dalla Rivoluzione d'Ottobre ${ }^{2}$. La

\footnotetext{
${ }^{1}$ Cfr. FOA, Vittorio, RICCIARDI, Andrea, «Dialogo breve sulla rivoluzione», in Annali della Fondazione Ugo La Malfa, XX, 2005, pp. 235-241, p. 235. Si chiedeva ancora Foa: «perché le rivoluzioni non riescono? Io penso che ci sia una ragione fondamentale. La rivoluzione mira "soltanto" a cambiare radicalmente la realtà, non ha in sé elementi per rendere stabili i mutamenti, non sa costruire come sa distruggere. Se non si dimostra di aver chiaro anche ciò che si vuole conservare, oltre a ciò che si vuole abbattere, si crea una situazione un po' delicata...Tutte le rivoluzioni, anche quando hanno ottenuto nel tempo risultati straordinari, come è stato per la Rivoluzione Francese o quella inglese, sono destinate a fallire rispetto agli obiettivi iniziali perché tendono a non fermarsi, arrivando addirittura a distruggersi». Ibidem, p. 238.

${ }^{2}$ Tra le pubblicazioni più recenti, mi limito a citare PETRACCHI, Giorgio (a cura di), «L'Italia e la rivoluzione d'ottobre. Masse, classi, ideologie, miti tra guerra e primo dopoguerra», in Annali della Fondazione Ugo La Malfa, XXXI, 2016, pp. 43-388, con contributi di Roberto Bianchi, Bedeschi, Cinnella, Olga Dubrovina, Andreucci, Strada, Santi Fedele, Bresciani, Antonello Venturi, Panaccione, Giulio Carpi, Pellicani, Garzonio, Breschi, Mariuzzo, Morozzo della Rocca, Petracchi; CARIOTI, Antonio (a cura di), Ottobre rosso. La rivoluzione che ha cambiato la storia, Milano, RCS, 2017, con testi di Allevato, Canfora, Carioti, Codevilla, Cremonesi, Dragosei, Flores, Galli della Loggia, Kolonitsky, Magarotto, Mettan, Mikhalkov, Montefiori, Moscato,
} 
politica attuale è quanto mai lontana dal Novecento, i partiti comunisti sono da tempo scomparsi dagli scenari nazionali e internazionali, con pochissime eccezioni, ma anche quelli socialisti e socialdemocratici non sembrano godere di buona salute. La stessa idea di sinistra, al di là delle sigle di partito e delle differenti matrici ideologico-culturali del movimento operaio sviluppatesi tra gli ultimi decenni dell'Ottocento e la fine della Guerra fredda, pare essere profondamente in crisi. Non è così per i populismi (categoria non semplice da definire e talvolta un po' abusata) e per la destra (soprattutto quella radicale), molto popolare tra i giovani, certamente più di quanto non si potesse immaginare soltanto qualche anno fa.

Sarebbe fuori luogo in questa sede provare a indagare i perché di questo scenario, per vari aspetti inquietante, che appare in linea con il crescente indebolimento dell'antifascismo su cui, almeno in Italia, sono state faticosamente edificate la Repubblica e la democrazia dopo il 1945, cioè in seguito alla sconfitta del nazifascismo e alla fine della Seconda guerra mondiale. Qui ci si propone solo di fornire qualche spunto di riflessione sul 1917 e, più precisamente, su come la Rivoluzione d'Ottobre è stata discussa in un denso volume collettaneo, ideato e curato da Marco Di Maggio3. Il libro, che ospita contributi di differente spessore scientifico, affronta un tema di notevole interesse: la lettura (quindi la ricezione e l'utilizzo politico) della Rivoluzione bolscevica da parte di partiti e movimenti italiani della sinistra marxista (il PCI di Togliatti, Longo e Berlinguer; il PSI di Nenni e, prima, di Turati e Serrati; il PSIUP di Vecchietti e Basso), ai quali si aggiungono tre riviste e giornali di tutt'altri contesti politico-culturali («La Civiltà Cattolica», «Corriere della Sera» e «La Stampa»); figure appartenenti all'area nazionalista, per lo più confluita nel fascismo; Giustizia e Libertà di Rosselli; il Movimento Sociale Italiano di Michelini e Almirante; la "Nuova Sinistra". Un ambito, quest'ultimo, più diversificato al suo interno di quanto non si possa ritenere a una lettura superficiale del fenomeno. Basti pensare alla grande distanza ideologica tra i vari gruppi nati nel corso degli anni Sessanta, più o meno ispirati allo stalinismo, al maoismo o a forme eterodosse di marxismo: Servire il Popolo, Programma comunista, Lotta comunista, i Quaderni Rossi (fondati nel 1961, in un contesto socio-culturale molto diverso da quello di fine decennio), Classe operaia, Potere operaio, Lotta continua e Il Manifesto, il meno settario e anche il più "fertile" e duraturo.

I saggi contenuti nel libro, come spiega Di Maggio nell'introduzione, sono organizzati seguendo tre scansioni temporali: la prima comprende gli scritti sulle letture della Rivoluzione nel periodo tra le due guerre, compiute, rispettivamente, dalle varie anime dell'ancora unito socialismo italiano (D'Alessandro); da esponenti del nazionalismo più o meno vicini a Mussolini,

Romano, Salomoni, Terekhova, Valentino.

${ }^{3}$ DI MAGGIO, Marco (a cura di), Sfumature di rosso. La Rivoluzione russa nella politica italiana del Novecento, Torino, Accademia University Press, 2017. Il volume fa parte della BHM (la Biblioteca della rivista «Historia Magistra»), collezione diretta da Angelo d'Orsi (qui autore della Postfazione), e presenta saggi di D’Alessandro, Cingari, Bufarale, Bucci, Ambrosi, Sorgonà, Chiarotto, Cappelli, Höbel, Strippoli e Di Maggio. 
tra cui Corradini, Rocco, Marinetti e Pantaleoni (Cingari); da Giustizia e Libertà, fondata nel 1929 a Parigi e, a sua volta, portatrice di una visione rivoluzionaria dell'antifascismo non omogenea, se si considerano le differenti posizioni emerse durante gli anni Trenta fra gli stessi dirigenti (Bufarale).

Il secondo gruppo di saggi affronta un arco temporale di più lunga durata: Bucci documenta le posizioni dell'organo dei gesuiti, «La Civiltà Cattolica», dal 1917 al 1991; Ambrosi tratta il modo in cui furono affrontati i decennali della Rivoluzione dal 1947 al 1987 nei due quotidiani, «Corriere della Sera» e «La Stampa», forse più autorevoli nell'esprimere le istanze delle classi dirigenti liberal-democratiche e liberal-conservatrici; Sorgonà analizza le posizioni emerse all'interno del mondo neofascista, dalla fondazione dell'MSI alla sua scomparsa negli anni Novanta.

Il terzo gruppo di scritti, il più corposo, si concentra sull'atteggiamento delle diverse culture politiche della sinistra marxista tra la parte finale della Seconda guerra mondiale e l'inizio del tramonto della cosiddetta Prima Repubblica, seguendo una successione cronologica. Sul periodo 1944-1954, Chiarotto e Capelli; sulla fase che va dal "terribile 1956" alla solidarietà nazionale, Höbel; sul "lungo" Sessantotto, Strippoli; sul periodo compreso tra il 1977 e la fine dell'URSS, Di Maggio. Quest'ultimo sembra voler mostrare non soltanto il definitivo distacco dal marxismoleninismo del PSI di Craxi durante gli anni Ottanta, ma anche il progressivo "sgretolamento" del legame dello stesso PCI con il leninismo e, dunque, con l'eredità della Rivoluzione d'Ottobre soprattutto dopo la scomparsa di Berlinguer, l'uomo della rottura (non totale) con Mosca.

Se si riflette sulla prima parte del volume, colpisce (ma non sorprende) come della Rivoluzione, in corso d'opera, si sapesse davvero poco. Questo elemento emerge a più riprese nel saggio di D'Alessandro che, per esempio, fa notare come lo stesso Gramsci, per buona parte del 1917, avesse individuato nel socialrivoluzionario Černov la figura più rappresentativa della rivoluzione, identificando «in lui il capo dei massimalisti russi proprio in contrappunto con la figura di Lenin $»^{4}$. Per quanto a fine luglio, a Firenze, la rottura interna alla Direzione socialista avesse fatto emergere la frattura tra le due anime del partito, anche fra gli intransigenti la conoscenza degli avvenimenti russi (inevitabilmente connessa con le strategie da adottare in Italia per "fare come in Russia") era tutt'altro che approfondita. Anche i riformisti, pur perplessi e preoccupati dalle notizie provenienti da Pietrogrado, «sembravano giustificare la Rivoluzione, quantomeno dal punto di vista storico, trovando cioè un riferimento nelle rivoluzioni del passato. La lettura della Rivoluzione data da Treves, come "cataclisma necessario in Natura", e quella data da Modigliani, come "tentativo comunardo disperato di salvare il proprio paese dalla rovina", rispondono sostanzialmente a questo approccio» ${ }^{5}$. Tra la fine del 1917 e l'inizio del 1918, in seguito allo scioglimento dell'Assemblea Costituente, mentre emergeva la distanza tra Gramsci e Bordiga

\footnotetext{
${ }^{4}$ Ibidem, p. 11.
}

${ }^{5}$ Ibidem, p. 21. 
sull'interpretazione della visione marxista della storia, anche Serrati, di fronte alla presa di distanza di Martov dai bolscevichi, difendeva il loro operato ed evocava "il dovere dei socialisti" di contribuire alla vittoria della Rivoluzione, evitando pericolose frammentazioni. Soltanto «Critica Sociale», con Treves, difendeva le posizioni del leader menscevico, senza tuttavia volersi staccare dalla dottrina marxista e confermando quanto, in quella fase di passaggio molto confusa, fosse ampia la distanza tra il socialismo riformista di marca turatiana e il revisionismo socialdemocratico che, in Italia, si sarebbe affermato solo in un secondo tempo.

Tra i nazionalisti, la Rivoluzione d'Ottobre venne declinata in modi diversi. Cingari, alludendo alle istanze espresse nel 1920 dall'irredentista triestino Attilio Tamaro, scrive che per lui «se il bolscevismo era dottrina da combattere e da respingere, non così era il sistema di governo dittatoriale, nato in mezzo alla tragedia, che andava considerato seriamente ed adottato anche in Europa Occidentale» ${ }^{6}$. Anche per Marinetti non era dunque la difesa dell'esistente il discrimine tra nazionalisti e comunisti, quanto gli effetti del processo rivoluzionario: in Italia era necessaria una rivoluzione nazionale dai connotati inegualitari e anarchico-individualisti che, tenendo fermo il valore della patria (diversamente declinato dai socialisti riformisti), non mirasse a livellare «i soggetti in una mediocre uguaglianza» ${ }^{7}$, come stava accadendo nella Russia bolscevica.

Non è certamente possibile, in questa sede, soffermarsi su tutti i saggi contenuti nel libro, ma solo dar conto brevemente della struttura o dei tratti salienti di alcuni di essi. Bufarale, per spiegare le analisi svolte dai giellisti sulla Rivoluzione d'Ottobre negli anni Trenta, prende in considerazione scritti di Rosselli (che nel 1934 cercò un'intesa con Trotskij e il gruppo di Tresso, Leonetti e Ravazzoli espulso dal PCd'I nel 1930), Nicola Chiaromonte, Silvio Trentin, Caffi, Lussu, Leone Ginzburg, Franco e Lionello Venturi. Bufarale, con la sua ricognizione, da una parte mostra la ricchezza del dibattito interno al movimento e, dall'altra, l'eterogeneità delle posizioni espresse che, non a caso, si tradussero nella rottura tra i "novatori" (Caffi e Chiaromonte) e il resto del nucleo dirigente.

Di particolare interesse, guardando al saggio su «La Civiltà Cattolica», appaiono le osservazioni di Bucci inerenti al primo editoriale del gennaio 1990, sottoscritto da tutta la redazione quando, dopo la caduta del Muro di Berlino (novembre 1989), l'URSS e il comunismo sembravano avviati al crollo definitivo. Nell'editoriale, per certi aspetti profetico, tra l'altro si leggeva:

Se l'Est piange, l'Ovest dell'Europa non ride. Certamente il capitalismo occidentale può vantare la sua vittoria sul comunismo orientale; ma [...] la stessa maggiore ricchezza prodotta non è equamente distribuita e la sua produzione si compie, almeno in una certa misura, a spese del Terzo Mondo, la cui situazione è assai più grave di quella dei Paesi dell'Est, ma che potrebbe subire, se non una

\footnotetext{
${ }^{6}$ Ibidem, p. 41.
}

${ }^{7}$ Ibidem, p. 45. 
dimenticanza, certo una riduzione degli aiuti da parte dei Paesi occidentali, impegnati ad aiutare con urgenza i Paesi dell'Est ${ }^{8}$.

Conclude Bucci:

«Civiltà Cattolica» si sofferma sul ritorno dell'idea di nazione, che presagisce lo sbriciolamento dell'Unione Sovietica, e rivolge quindi il suo sguardo ad Occidente, annunciando un più complesso campo di confronto: concluso lo scontro ideologico e religioso con il comunismo, come confrontarsi con le contraddizioni del «mondo libero»? Come affrontare la sempre più vicina riunificazione tedesca, che ricostruisce una Germania forte nello spazio di un'Europa in cerca d'identità? In tali pagine, apprezziamo la capacità dei gesuiti di precorrere molte domande della contemporaneità [...]. La rivista dimostra massima consapevolezza circa i temi nuovi che attendono i cattolici: la ricostruzione di un ruolo pubblico per il cristianesimo nell'Europa orientale che affronta un percorso di democratizzazione, il rischio di un Occidente cieco che accoglie l'Est solo per ricavarne vantaggi ${ }^{9}$.

Sono passati quasi trent'anni dal crollo dell'URSS e cento dalla Rivoluzione d'Ottobre, che ne costituì la radice. Forse sono pochi ad avvertire la nostalgia della rivoluzione, sulla quale vale la pena di interrogarsi ancora (magari contemplando anche le elaborazioni sul tema da parte delle altre famiglie politiche), ma quasi tutti sanno bene che le risposte date finora alle domande di sviluppo e giustizia sociale dell'epoca contemporanea sono insufficienti e che il nuovo mondo globale, in cui di rivoluzione quasi non si discute, non sembra aver risolto i vecchi problemi emersi con forza durante la Grande guerra e nell'immediato primo dopoguerra, non solo in Russia.

\footnotetext{
${ }^{8}$ Ibidem, p. 99.

${ }^{9}$ Ibidem.
} 


\section{L'AUTORE}

Andrea RICCIARDI collabora dal 2000 con l'Università degli Studi di Milano dove si è laureato, ha conseguito il dottorato di ricerca in Storia della società e delle istituzioni nell'Europa contemporanea, ha lavorato per quattro anni come assegnista ed è docente a contratto di Storia contemporanea. Si occupa di storia politica e culturale, con particolare riferimento a vicende e figure inerenti alla storia del movimento operaio e dell'antifascismo. Dal 2010 è membro della redazione de «Il Mestiere di Storico» e dal 2011 del Comitato di direzione degli «Annali della Fondazione Ugo La Malfa». Nel 2013 ha ottenuto l'ASN per professore associato di Storia contemporanea. Oltre a vari saggi pubblicati in riviste di settore e in volumi collettanei, ha scritto e curato libri su Leo Valiani, Vittorio Foa, Gino Giugni, Riccardo Lombardi, Piero Boni, Antonio Giolitti e Luigi Longo.

URL: $<$ http://www.studistorici.com/progett/autori/\#Ricciardi > 